\title{
FOUR DIMENSIONAL COMPACT LOCALLY HERMITIAN MANIFOLDS
}

\author{
BY \\ LOUIS AUSLANDER( ${ }^{(1)}$
}

Introduction. The purpose of this paper is to classify the compact real four dimensional locally hermitian manifolds. A manifold is called locally hermitian if it is a complex manifold with an hermitian metric with curvature and torsion equal to zero. In $\S 1$, we discuss the general theorems which we will use in the rest of the paper. $\$ 2$ is devoted to proving that there are only five possible holonomy groups $H_{1}, \cdots, H_{5}$. These are cyclic groups of orders $1,2,3,4$ and 6 respectively. At the beginning of $\$ 3$ we outline the steps we will follow in computing the allowable group extensions. There then follow five steps in which we give the details of this outline. The number of group extensions for a given holonomy group is given by the following table:

\begin{tabular}{l|c|c|c|c|c} 
Holonomy groups & $H_{1}$ & $H_{2}$ & $H_{3}$ & $H_{4}$ & $H_{5}$ \\
\hline Number of extensions & 1 & 2 & 2 & 2 & 1
\end{tabular}

We have added an appendix in which we discuss the topology of compact locally hermitian manifolds. We have shown that there are only two possible Poincaré polynomials for four dimensional compact locally hermitian manifolds $1+2 x+2 x^{2}+2 x^{3}+x^{4}$ and $1+4 x+6 x^{2}+4 x^{3}+x^{4}$. We have further shown that only the torus has Poincare polynomial $1+4 x+6 x^{2}+4 x^{3}+x^{4}$. It should be remarked that the discussion in this appendix could be used to give an alternative proof for Theorem 5 .

1. General considerations. Let $M$ be a complete $n$-dimensional riemann manifold with curvature and torsion equal to zero. We will call such spaces locally euclidean spaces. Then, if $M I^{*}$ denotes the universal covering space of $M$ with its induced riemann metric, it is clear from [2] that $M^{*}$ is isometric to $n$-dimensional euclidean space $E^{n}$. We will identify $M^{*}$ and $E^{n}$ by means of this isometry. The fundamental group $\pi$ of $M$ acting on $E^{n}$ preserves the euclidean structure in $E^{n}$ and may therefore be considered as a subgroup of the group $R(n)$ of all rigid motions of $E^{n}$. Further, $M$ is homeomorphic to the orbit space of $E^{n}$ under $\pi$. We will denote this orbit space by $E^{n} / \pi$. It is also easy to see that if $\pi \subset R(n)$ and $E^{n} / \pi$ is an $n$-dimensional manifold then it is a locally euclidean space. Hence we see that the study of locally euclidean spaces is equivalent to the study of those discrete subgroups $\pi$ of $R(n)$ such that $E^{n} / \pi$ is an $n$-dimensional manifold.

Received by the editors March 10, 1956 and, in revised form, May 6, 1956.

(1) This paper was written while the author was a National Science Post-Doctoral Fellow. 
Let $C^{n}$ be the $n$-dimensional complex vector space with coordinates $\left(z_{1}, \cdots, z_{n}\right)$. Let $R(n, C)$ be the group of all linear transformations and translations which preserve the quadratic form $\sum_{j=1}^{n} z_{j} \bar{z}_{j}$. Then the same discussion as used in the locally euclidean case can be used to show that $M$ is an $n$ complex dimension locally hermitian manifold if and only if there exists an imbedding of the fundamental group $\pi$ of $M$ into $R(n, C)$ such that $M$ is homeomorphic to $C^{n} / \pi$.

We will for the rest of this paper restrict our attention to compact manifolds. The following is a list of several theorems from the theory of compact locally euclidean spaces which we will need. Proofs of Theorems 1, 2 and 4 are to be found in [3] and [4]. Theorem 3 is proved in [1].

THEOREM 1. Let $N$ denote the subgroup of $\pi$ consisting of pure translations. Then $N$ is generated by $n$ linearly independent translations and $\pi / N$ is a finite group.

Definition. We will denote $\pi / N$ by $h(\pi)$ and call it the holonomy group of $\pi$.

Let the order of $h(\pi)$ be $\rho$ and let $\xi \in \pi$. Then $\xi^{\rho} \in N$. We will denote $\xi \in R(n)$ by $(\eta, T)$, where $\eta$ is an element of the $n$-dimensional orthogonal group $O(n)$ and $T$ is a vector in $E^{n}$. We will consider $O(n)$ and $E^{n}$ imbedded in $R(n)$ in the obvious way. It should be noted that we may consider $h(\pi)$ imbedded in $O(n)$. We will make this identification whenever it is convenient. We will of ten let $T=(e, T)$ and since $T_{1} T_{2}=\left(e, T_{1}\right)\left(e, T_{2}\right)=\left(e, T_{1}+T_{2}\right)$, we will use additive notation for the law of composition of translations.

Theorem 2. Let $T_{1}, \cdots, T_{n}$ be a basis for $N$. Then $h(\pi)$ written in terms of the basis $T_{1}, \cdots, T_{n}$ is a matrix group over the ring of integers.

THEOREM 3. Let $\pi$ be a subgroup of $R(n)$ with the following properties:

1. The subgroup $N$ of $\pi$ consisting of pure translations is generated by $n$ linearly independent translations.

2. $\pi / N$ is finite.

3. $\pi$ contains no finite subgroups. Then $E^{n} / \pi$ is a compact locally euclidean space.

Let $A(n)$ denote the group of all affine motions of $E^{n}$, i.e. all linear transformations and translations.

THEOREM 4. Let $\pi$ and $\pi^{\prime}$ be isomorphic subgroups of $R(n)$ such that $E^{n} / \pi$ and $E^{n} / \pi^{\prime}$ are $n$-dimensional compact manifolds. Then there exists a $g \in A(n)$ such that $g^{-1} \pi g=\pi^{\prime}$.

COROLlary 1. Let $\pi$ and $\pi^{\prime}$ be subgroups of $R(n)$ such that $E^{n} / \pi$ and $E^{n} / \pi^{\prime}$ are compact manifolds. Assume $h(\pi)$ and $h\left(\pi^{\prime}\right)$ are identical subgroups of $O(n)$. Then if $N$ and $N^{\prime}$ denote the group of translations in $\pi$ and $\pi^{\prime}$ respectively, $\pi$ is isomorphic to $\pi^{\prime}$ only if there is a mapping of $E^{n}$ onto itself which maps $N$ onto 
$N^{\prime}$ and the induced mapping on $O(n)$ leaves $h(\pi)$ invariant (not necessarily pointwise).

Let $\pi$ and $\pi^{\prime}$ be subgroups of $R(n)$ such that $E^{n} / \pi$ and $E^{n} / \pi^{\prime}$ are compact locally euclidean manifolds. Assume further that $N=N^{\prime}$ and $h(\pi)=h\left(\pi^{\prime}\right)$ as subgroups of $O(n)$.

COROLlaRY 2. $\pi$ is isomorphic to $\pi^{\prime}$ if and only if we may choose two bases $T_{1}, \cdots, T_{n}$ and $T_{1}^{\prime}, \cdots, T_{n}^{\prime}$ of $N$ and preimages of $h(\pi), I(\pi)$ and $I\left(\pi^{\prime}\right)$ in $\pi$ and $\pi^{\prime}$ respectively, such that:

1. If we let $h(\pi)$ act on $N$ by $\eta^{-1} T \eta$ for $\eta \in h(\pi)$ and $T \in N$, then the mapping $A$ of $N$ onto itself determined by $A\left(T_{i}\right)=T_{i}^{\prime}, i=1, \cdots, n$ is invariant under the action of $h(\pi)$.

2. If $\eta_{1}$ and $\eta_{2}$ have counterimages $\xi_{1}$ and $\xi_{2}$ in $I(\pi)$ and $\xi_{1}^{\prime}$ and $\xi_{2}^{\prime}$ in $I\left(\pi^{\prime}\right)$ respectively and $\xi_{1} \xi_{2} \in N$, then $A\left(\xi_{1} \xi_{2}\right)=\xi_{1}^{\prime} \xi_{2}^{\prime}$.

Proof. The necessity of these conditions is clear. To prove the conditions are sufficient, we note that every element of $\pi$ and $\pi^{\prime}$ can be written in one and only one way as $T_{1}^{i_{1}} \cdots T_{n}^{i_{n} \xi}$ and $A\left(T_{1}^{i_{1}} \cdots T_{n}^{i_{n}}\right) \xi^{\prime}$ for $\xi \in I(\pi)$ and $\xi^{\prime} \in I\left(\pi^{\prime}\right)$. Now map $I(\pi)$ onto $I\left(\pi^{\prime}\right)$ by corresponding those elements which map into the same element of $h(\pi)$. Now this mapping of $I(\pi)$ onto $I\left(\pi^{\prime}\right)$ and $N$ onto $N^{\prime}$ gives us a mapping $A^{*}$ of $\pi$ onto $\pi^{\prime}$. Conditions 1 and 2 assure us that $A^{*}$ is a homomorphism from which it is trivial to see that $A^{*}$ is actually an isomorphism of $\pi$ onto $\pi^{\prime}$.

2. On $h(\pi)$. We will henceforth restrict our attention to compact two complex dimensional, four real dimensional, hermitian manifolds. We will say that two such manifolds are similar if $\pi$ and $\pi^{\prime}$ are isomorphic or, what amounts to the same thing, if once we imbed $R(2, C)$ in $R(4)$ there exists a $g \in A(4)$ such that $g^{-1} \pi g=\pi^{\prime}$. We propose to classify the compact 2 complex dimensional hermitian manifolds up to similarity.

THEOREM 5. We may choose a basis for $C^{2}$ in terms of which every element $\eta$ of $h(\pi)$ has the form

$$
\eta=\left(\begin{array}{ll}
e^{i \theta} & 0 \\
0 & 1
\end{array}\right)
$$

$\operatorname{Proof}\left({ }^{2}\right)$. Let $\left(z_{1}, z_{2}\right)$ be a coordinate system for $C^{2}$ and let $R(2, C)$ be written as

$$
z^{\prime}=a_{\alpha 1} z_{1}+a_{\alpha 2} z_{2}+c_{\alpha} \quad(\alpha, \beta=1,2),
$$

where $\left(a_{\alpha \beta}\right)$ is a unitary matrix and, except for the identity transformation no point in $C^{2}$ is fixed under any transformation of the group. From this and Cramer's rule it is easy to see that each unitary matrix $\left(a_{\alpha \beta}\right)$ has at least one

(2) The following proof for this theorem was suggested by the referee. 
eigenvalue equal to one. Let $\Theta_{i}(i=1,2)$ be two unitary matrices of the type in question, both different from the identity. Under suitable choice of unitary basis for $C^{2}$ these matrices have the form

$$
\begin{aligned}
& \Theta_{1}=\left(\begin{array}{ll}
e^{i \theta_{1}} & 0 \\
0 & 1
\end{array}\right) \\
& \Theta_{2}=\left(\begin{array}{ll}
a & b \\
c & d
\end{array}\right) .
\end{aligned}
$$

The condition that both $\Theta_{2}$ and $\Theta_{1} \Theta_{2}$ have an eigenvalue one gives the following equations:

$$
\begin{aligned}
(a-1)(d-1)-b c & =0, \\
\left(a e^{i \theta_{1}}-1\right)(d-1)-b c e^{i \theta_{1}} & =0 .
\end{aligned}
$$

Multiplying the first equation by $e^{i \theta}$ and subtracting it from the second gives

$$
\left(e^{i \theta_{1}}-1\right)(d-1)=0 .
$$

Since $e^{i \theta_{1}} \neq 1$, this implies that $d=1$, and since $\Theta_{2}$ is unitary, we must have

$$
\Theta_{2}=\left(\begin{array}{ll}
c^{i \theta_{2}} & 0 \\
0 & 1
\end{array}\right)
$$

COROllary 3. $h(\pi)$ is a finite cyclic group.

We will henceforth exclude the case $H_{1}$, where $h(\pi)$ consists of the identity element only.

Lemma 1. Let $\xi=(\eta, R+S)$, where $R$ is contained in the invariant space of $\eta$ and $S$ is in the orthogonal complement. Then $\xi^{\rho}=(e, \rho R)$.

Proof. By a straightforward calculation, we have $\xi^{\rho}=(e, T)$, where

$$
T=\rho R+\left(\Theta^{\rho-1} S+\cdots+\Theta S+S\right)
$$

and

$$
\Theta=\left(\begin{array}{rr}
\cos \theta & \sin \theta \\
-\sin \theta & \cos \theta
\end{array}\right)
$$

Now $\Theta^{p-1} S+\cdots+S=0$, since it is the sum of the vectors from the center of a regular plane polygon to the vertices of the polygon.

Lemma 2. Let $R^{*}+S^{*} \in N$, where $R^{*}$ is in the invariant space of $\eta$ and $S^{*}$ is in the orthogonal complement. Then $\rho R^{*}$ and $\rho S^{*}$ are elements of $N$.

Proof. I.et $\xi \in \pi$ such that $\xi=(\eta, R+S)$. Then

$$
\left(R^{*}+S^{*}\right) \xi=\left(\eta,\left(R+R^{*}\right)+\left(S+S^{*}\right)\right) .
$$


Hence by applying Lemma 1 we have $\rho R^{*} \in N$ and hence $\rho S^{*} \in N$.

Now let $T_{i}=R_{i}+S_{i}, i=1, \cdots, 4$ be a basis for $N$. Then $\rho S_{i} \in N$, $i=1, \cdots, 4$. Assume $\rho S_{1}$ and $\rho S_{2}$ span the space $x_{3}=x_{4}=0$. Then by Theorem $1, \Theta$ must be a matrix with rational entries relative to the bases $S_{1}$ and $S_{2}$. Hence $\Theta$ must be similar to a matrix with integer entries. But, since $\lambda^{2}-2 \cos \theta \lambda+1$ is the characteristic equation of $\Theta$, its coefficients must be integers. Hence $2 \cos \theta=0, \pm 1, \pm 2$. We have therefore proved

THEOREM 6. $h(\pi)$ can be cyclic groups of order 1, 2, 3, 4 and 6 only.

3. On the extension problem. We will now outline the remaining steps in the classification problem before presenting the details. We have already in $\$ 2$ determined the possible holonomy groups. The construction of the possible holonomy groups then is reduced to a group extension problem with the added conditions that $\pi$ must be imbedded in $R(4)$ and $E^{4} / \pi$ must be a compact manifold. We will use the following steps to determine the groups $\pi$ up to similarity:

SteP 1. Take a fixed basis in $E^{4}$ as determined in Theorem 5 and a fixed representation for $h(\pi)$. Let $N$ be a subgroup of $R(4)$ generated by 4 linearly independent translations. Then the fact that $h(\pi)$ must be an integer matrix group over any basis for $N$ assures us that we may choose a basis for $N$ satisfying certain conditions. These conditions are given in Lemmas 3 and 4 .

STEP 2. By picking a new basis for $N$ if necessary we show that we may always assume $N$ is generated by a basis of one of the following types:

$$
\begin{array}{ll}
\text { type (a) } S_{1}+(k / \rho) R_{3}, S_{2}, R_{3}, R_{4}, & 0 \leqq k<\rho, \\
\text { type (b) } S_{1}+\frac{1}{2} R_{3}, S_{2}+\frac{1}{2} R_{4}, R_{3}, R_{4} &
\end{array}
$$

where $R_{3}$ and $R_{4}$ lie in the invariant space of $h(\pi), S_{1}$ and $S_{2}$ in the orthogonal complement to the space spanned by $R_{1}$ and $R_{2}$ and $\rho$ equals the order of $h(\pi)$. The only time type $b$ occurs is when $h(\pi)$ is of order 2 . These will be called a canonical basis for $N$ of type a and b respectively. Two canonical forms of type a for a given $\rho$ will be called the same only if the $k$ is the same in both of them.

STEP 3. For each $h(\pi)$ and $N$ the group extension $\pi$ of $N$ by $h(\pi)$ is determined by the choice of $T$ in $\xi=(\eta, T)$, where $\eta$ is a generator of $h(\pi)$. The requirement that $E^{4} / \pi$ should be a manifold then shows that only canonical basis of type a are possible. Further all $N$ with canonical basis of type a have an allowable extension given by taking $T=(1 / \rho) R_{4}$, where $\rho$ equals the order of $h(\pi)$.

STEP 4. Up to a new choice of canonical basis of type a for $N$ and a generator for $h(\pi)$, the above extension is the only possible extension for a given canonical basis of type a. 
Step 5. Let $\pi$ and $\pi^{\prime}$ be extensions of $N$ and $N^{\prime}$ by $h(\pi)$ where $N$ and $N^{\prime}$ have canonical bases of type a given by

$$
\begin{aligned}
& S_{1}+(k / \rho) R_{3}, S_{2}, R_{3}, R_{4}, \\
& S_{1}^{\prime}+\left(k^{\prime} / \rho\right) R_{3}^{\prime}, S_{2}^{\prime}, R_{3}^{\prime}, R_{4}^{\prime} .
\end{aligned}
$$

Then $\pi$ is similar to $\pi^{\prime}$ if and only if $k= \pm k^{\prime} \bmod (\rho)$.

STEP 1 . We may actually assume more about $S_{1}$ and $S_{2}$ than we stated in paragraph 2. We may assume further that $S_{3}$ and $S_{4}$ can be written as an integer linear combination of $S_{1}$ and $S_{2}$. This follows from the fact that $\rho S_{i}, i=1, \cdots, 4$, lie in a two-dimensional space and since $\pi$ acts without fixed points or accumulation points, all of them can be written as an integer linear combination of two of them. We may call these two $S_{1}$ and $S_{2}$. Hence we may choose a basis for $N$ of the form

$$
R_{1}+S_{1}, R_{2}+S_{2}, R_{3}, R_{4} \text {. }
$$

Lemma 3. $R_{3}$ and $R_{4}$ span the invariant space of $\eta$.

Proof. $\rho S_{1}$ and $\rho S_{2}$ are elements of $N$. Since $S_{1}$ and $S_{2}$ are linearly independent

$$
\begin{aligned}
& -\rho S_{1}=-\rho R_{1}-\rho S_{1}+a_{13} R_{3}+a_{14} R_{4}, \\
& -\rho S_{2}=-\rho R_{2}-\rho S_{2}+a_{23} R_{3}+a_{24} R_{4}
\end{aligned}
$$

or

$$
\begin{aligned}
& \rho R_{1}=a_{13} R_{3}+a_{14} R_{4}, \\
& \rho R_{2}=a_{23} R_{3}+a_{24} R_{4}
\end{aligned}
$$

where $a_{13}, a_{14}, a_{23}, a_{24}$ are integers. But $R_{i}, i=1, \cdots, 4$ spans the invariant space of $\eta$. This proves the lemma.

Now $R_{1}+\Theta S_{1}$ and $R_{2}+\Theta S_{2}$ are elements of $N$. Let $\left(\eta_{i j}\right), i, j=1,2$, be the matrix which represents $\Theta$ in terms of the bases $S_{1}$ and $S_{2}$. Then

$$
R_{i}+\Theta S_{i}=\sum \eta_{i j} S_{j}+\sum \eta_{i j} R_{j}+\sum b_{i \alpha} R_{\alpha},
$$

where $i, j=1,2, \alpha=3,4$ and $b_{i \alpha}$ are integers. But $R_{i}=\sum\left(a_{i \alpha} / \rho\right) R_{\alpha}$.

Lemma 4. $(1 / \rho)\left(a_{i \alpha}-\sum \eta_{i j} a_{j \alpha}\right)$ must be integers.

STEP 2 . We may clearly always assume that for each case $0 \leqq a_{i \alpha}<\rho$.

CASE $\rho=2$. For this case

$$
\left(\eta_{i \alpha}\right)=\left(\begin{array}{rr}
-1 & 0 \\
0 & -1
\end{array}\right)
$$

and then the formula of Lemma 4 gives 


$$
\left(\begin{array}{ll}
a_{13} & a_{14} \\
a_{23} & a_{24}
\end{array}\right) .
$$

Hence any values of 0 or 1 are admissible for $a_{13}, a_{14}, a_{23}, a_{24}$. We will discuss completely the case where all $a_{i \alpha}=1$ and explicitly reduce this to a canonical basis. We will then state the other results without discussion since the technique for carrying these discussions out will have been given once in detail.

We have a basis of $N$ of the form

$$
S_{1}+\frac{1}{2} R_{3}+\frac{1}{2} R_{4}, \quad S_{2}+\frac{1}{2} R_{3}+\frac{1}{2} R_{4}, R_{3}, R_{4} .
$$

Let $R_{3}^{\prime}=R_{3}+R_{4}, R_{4}^{\prime}=R_{3}-R_{4}$. Then clearly

$$
S_{1}+\frac{1}{2} R_{3}^{\prime}, \quad S_{2}+\frac{1}{2} R_{3}^{\prime}, R_{3}^{\prime}, R_{4}^{\prime}
$$

is a basis for $N$. Now consider

$$
S_{1}+\frac{1}{2} R_{3}^{\prime}, \quad S_{1}-S_{2}, R_{3}^{\prime} R_{4}^{\prime} .
$$

This is also a basis for $N$ and of canonical type a. The possible canonical bases are

$$
S_{1}+\frac{k}{\rho} R_{3}, S_{2}, R_{3}, R_{4}
$$

where $k=0$ or 1 and a canonical basis of type $\mathrm{b}$.

CASE $\rho=3$.

$$
\left(\eta_{i \alpha}\right)=\left(\begin{array}{rr}
0 & 1 \\
-1 & -1
\end{array}\right)
$$

and the formula of Lemma 4 gives

$$
\left(\begin{array}{cc}
\frac{a_{13}-a_{23}}{3} & \frac{a_{14}-a_{24}}{3} \\
\frac{2 a_{23}+a_{13}}{3} & \frac{2 a_{24}+a_{14}}{3}
\end{array}\right) \text { must be an integer matrix. }
$$

From this it can be shown that all canonical bases are of type a with $k=0,1$ or 2 .

CASE $\rho=4$.

$$
\left(\eta_{i \alpha}\right)=\left(\begin{array}{rr}
0 & -1 \\
1 & 0
\end{array}\right)
$$


and we have

$$
\left(\begin{array}{cc}
\frac{a_{13}+a_{23}}{4} & \frac{a_{14}+a_{24}}{4} \\
\frac{-a_{13}+a_{23}}{4} & \frac{-a_{14}+a_{24}}{4}
\end{array}\right)
$$

must be a matrix over the integers. This gives that all canonical bases are of type a with $k=0$ or 2 .

CASE $\rho=6$.

$$
\left(\eta_{i \alpha}\right)=\left(\begin{array}{rr}
0 & 1 \\
-1 & 1
\end{array}\right)
$$

and

$$
\left(\begin{array}{cc}
\frac{a_{13}+a_{23}}{6} & \frac{a_{14}+a_{24}}{6} \\
\frac{a_{13}}{6} & \frac{a_{14}}{6}
\end{array}\right)
$$

must be a matrix over the integers. This gives a canonical basis of type a with $k=0$.

We will summarize these results as a lemma.

LEMMA 5. The number of distinct canonical bases of type a are given by the following table

\begin{tabular}{c|c|c|c|c}
$\rho$ & 2 & 3 & 4 & 6 \\
\hline Canonical basis & 2 & 3 & 2 & 1
\end{tabular}

STEP 3. Now for each $h(\pi)$ and associated $N$, the group extension $\pi$, is determined by the choice of $T$ in $\xi=(\eta, T) \in \pi$, where $\eta$ is a generator of $h(\pi)$.

THEOREM 7. For a given $h(\pi)$ and associated $N$ a necessary and sufficient condition for a group extension $\pi$ to exist such that $E^{n} / \pi$ is a manifold is that we may choose $T$ such that:

1. $T=(1 / \rho)\left(a R_{3}+b R_{4}\right)$, where $a$ and $b$ are integers and $0 \leqq a \leqq b<\rho$.

2. $k T$ can be written as an integer linear combination of $R_{i}, i=1, \cdots, 4$, only if $k$ is divisible by $\rho$.

Proof. Assume a $T$ exists which satisfies the above conditions. We will then show that $\pi$ satisfies the hypothesis of Theorem 3. Now $\xi^{\rho}=a R_{3}+b R_{4}$ $\in N$. Hence hypotheses 1 and 2 are verified. To verify that there are no finite subgroups we note that 


$$
\left(T_{0} \xi\right)^{k}=\left(\eta^{k}, \eta^{k-1}\left(T+T_{0}\right)+\cdots+\eta\left(T+T_{0}\right)+T+T_{0}\right)
$$

for $T_{0} \in N$ and some $k$. Hence for this to be the identity element we must have that $\eta^{k}$ is the identity matrix or

$$
\eta^{k-1}\left(T+T_{0}\right)+\cdots+\left(T+T_{0}\right)=k\left(R+R_{0}\right),
$$

where $R+R_{0}$ is the perpendicular projection of $T+T_{0}$ into the invariant space of $\eta$. Hence this is zero only if $R+R_{0}=0$. But this is not possible by 2 . We must further verify that $\left(T_{0} \xi^{s}\right)^{k}$ does not equal the identity unless $T_{0} \xi^{s}$ is the identity. But this follows exactly as for $s=1$.

Now assume that the group $\pi$ with the desired properties exists. Then let $\eta$ be a generator of $h(\pi)$ and let $\xi=(\eta, R+S)$ be an element of $\pi$, where $R$ is contained in the covariant space of $\eta$ and $S$ is in the orthogonal complement. Then changing the origin of $E^{4}$ gives

$$
(e,-B)(\eta, R+S)(e, B)=(\eta, \eta B-B+S+R) .
$$

Hence we may choose $B$ in the orthogonal complement of the invariant space of $\eta$ and satisfying the equation $\eta B-B+S=0$. Hence in terms of the new origin $\xi=(\eta, R)$, where by our choice of bases for $N$

$$
R=(1 / \rho)\left(a R_{3}+b R_{4}\right) .
$$

Now since $\xi^{k}=\left(\eta^{k}, k R\right)$ we must have that $k R$ cannot be written as an integer linear combination of $R_{i}, i=1, \cdots, 4$. For otherwise, we would have a finite subgroup of $\pi$ which is impossible. We may by multiplying by a proper linear combination of $R_{3}$ and $R_{4}$ further assume that $0 \leqq a, b<\rho$. Further by relabeling, if necessary, we may assume $a \leqq b$. This completes the proof of this theorem.

Corollary. A basis of type a gives rise to permissible group extensions. But no basis of type $\mathrm{b}$ is allowable.

\section{STEP 4.}

LemMA 6. For a fixed $h(\pi)$ and $N$ of type a, with $k=0$, the group extension is unique up to similarity.

Proof. We first note that $T=1 / \rho R_{4}$ satisfies the hypothesis of Theorem 5 . Now let $T=(1 / \rho)\left(a R_{3}+b R_{4}\right)$ also satisfy hypothesis of Theorem 5 . By Corollary 2 we will prove our lemma once we have shown that the mapping $A^{*}$ which maps $a R_{3}+b R_{4}$ onto $R_{4}$ can be extended to a mapping $A$ of $N$ onto itself which leaves $h(\pi)$ invariant.

CASE 1. If $a$ and $b$ are relatively prime, then there exist integers $c$ and $d$ such that $a d-c b=1$. Hence we may define the mapping $A$ by $A\left(S_{1}\right)=S_{1}$, $A\left(S_{2}\right)=S_{2}, A\left(c R_{3}+d R_{4}\right)=R_{3}$ and $A\left(a R_{3}+b R_{4}\right)=R_{4}$. This mapping $A$ is of the desired type since all its entries are integers relative to $S_{1}, S_{2}, R_{3}, R_{4}$ and it has determinant one. 
CASE 2. Assume $a$ or $b=0$. Say $a=0$. Then by condition 2 of Theorem 5 , $b$ and $\rho$ must be relatively prime. Hence there exist $c$ and $d$ such that $c d-d \rho$ $=1$. Hence $c$ is relatively prime to $\rho$ or $\eta^{c}$ is a generator of $h(\pi)$. Hence $\xi^{c} \cdot R_{4}^{-d}$ may be used to replace $\xi$ and for it we have $T=(1 / \rho) R_{4}$.

CASE 3. Assume $a$ and $b$ are not relatively prime, satisfy the hypothesis of Theorem 5 , and $a$ and $b \neq 0$. Then it is easy to see that $a$ must equal $b$. Then we may map $R_{3}+R_{4} \rightarrow R_{4}$ and $R_{3}$ into $R_{3}$ leaving $S_{1}$ and $S_{2}$ invariant. Then Case 3 reduces to Case 2 which has already been treated.

Lemma 7. For a fixed $h(\pi)$ and $N$ of type a with $k \neq 0$ exactly one group extension is possible up to similarity.

Proof. Case 1. $N$ has a basis of the form

$$
S_{1}+(1 / \rho) R_{3}, S_{2}, R_{3}, R_{4} \text {. }
$$

Let $T=(1 / \rho)\left(a R_{3}+b R_{4}\right), a \neq 0$. For if $a=0$, this reduces to case 2 of previous lemma. Form $\left(S_{1}+(1 / \rho) R_{3}\right)^{-\rho \xi}=\xi^{\prime}$. We may choose this as a new element of $\pi$ such that modulo $N$ it is a generator of $h(\pi)$. But for $\xi^{\prime}, T=(b / \rho) R_{4}$. We may therefore apply case 2 of Lemma 6 if $b \neq 1$.

CASE 2. $N$ has a basis of the form

$$
S_{1}+(2 / \rho) R_{3}, S_{2}, R_{3}, R_{4} \text {. }
$$

L.et $T=(1 / \rho)\left(a R_{3}+b R_{4}\right)$. If $a=2$, then we may choose $\xi^{\prime}=\left(\eta, T^{\prime}\right)$ such that $T^{\prime}=(b / \rho) R_{4}-a S_{1}$. But by changing the origin, as we saw in Theorem 7 , we may assume $T^{\prime}=(b / \rho) R_{4}$. We again apply case 2 of Lemma 6 .

Since the above basis holds only for $\rho=3$ or 4 , we can always assume that if $a \neq 2$, then it equals 1 . Hence $b=1$ or 2 for $\rho=3$ and $b=1$ or 3 for $\rho=4$. For if $b=2$ for $\rho=4$, we would have $\left(S_{1}+R_{3} / 2\right)^{-1} R_{4}^{-1} \xi^{2}=\left(\xi^{2}, S_{1}\right)$, which is impossible. For $b=1$, we may define the mapping $A$ by $A\left(S_{1}+R_{3} / 2\right)=S_{1}+R_{3} / 2$, $A\left(S_{2}\right)=S_{2}, A\left(R_{3}\right)=R_{3}, A\left(R_{3}+R_{4}\right)=R_{4}$. But for $b=2$ or 3 in case $\rho=3$ or 4 respectively, we may replace $T$ by $(1 / \rho)\left(R_{3}-R_{4}\right)$. We may now define $A$ by

$$
\begin{aligned}
& A\left(S_{1}+R_{3} / 2\right)=S_{1}+R_{3} / 2, \\
& A\left(S_{2}\right)=S_{2}, \quad A\left(R_{3}\right)=R_{3} \\
& A\left(R_{3}-R_{4}\right)=R_{4} .
\end{aligned}
$$

This completes the proof of the lemma.

\section{STEP 5.}

We will prove at this step the following lemma.

Lemma 8. If $\pi$ and $\pi^{\prime}$ are extensions of $N$ and $N^{\prime}$ by $h(\pi)$ where $N$ and $N^{\prime}$ have canonical bases

$$
\begin{gathered}
S_{1}+(k / \rho) R_{3}, S_{2}, R_{3}, R_{4}, \\
S_{1}^{\prime}+\left(k^{\prime} / \rho\right) R_{3}^{\prime}, S_{2}^{\prime}, R_{3}^{\prime}, R_{4}^{\prime} .
\end{gathered}
$$


Then $\pi$ is similar to $\pi^{\prime}$ if and only if $k= \pm k^{\prime} \bmod (\rho)$.

We will first show that if $k=k^{\prime}$ then $\pi$ and $\pi^{\prime}$ are isomorphic. Assume $k=k^{\prime}=0$. Let $\eta$ be a generator of $h(\pi)$ and let $\eta$ written in terms of $S_{1}$ and $S_{2}$ be the matrix $\left(\eta_{i j}\right), i, j=1,2$. Then choose a new basis for $N^{\prime}$ by replacing $S_{1}^{\prime}$ and $S_{2}^{\prime}$ by $S_{1}^{*}$ and $S_{2}^{*}$ where $\eta$ in terms of the bases $S_{1}^{*}$ and $S_{2}^{*}$ is also the matrix $\left(\eta_{i j}\right)$. Clearly then the mapping which sends $S_{1}$ to $S_{1}^{*}, S_{2}$ to $S_{2}^{*}, R_{3}$ to $R_{3}^{\prime}$ and $R_{4}$ to $R_{4}^{\prime}$ gives an isomorphism of $\pi$ onto $\pi^{\prime}$.

Now if $k \neq 0$ and $k=k^{\prime}, \rho S_{1}$ and $S_{2}$ generate $N$ in the space spanned by $S_{1}$ and $S_{2}$. Then choose a new basis for $N^{\prime}$ by replacing $S_{1}^{\prime}+(k / \rho) R_{3}^{\prime}, S_{2}^{\prime}$ by $S_{1}^{*}+(k / \rho) R_{3}^{\prime}, S_{2}^{*}$ where $\rho S_{1}^{*}, S_{2}^{*}$ is also a basis for the space spanned by $\rho S_{1}^{\prime}$ and $S_{2}^{\prime}$ and where $\rho S_{1}^{*}, S_{2}^{*}$ has the further property that the matrix which expresses $\eta$ in terms of $\rho S_{1}$ and $S_{2}$ is the same as that which expresses $\eta$ in terms of $\rho S_{1}^{*}$ and $S_{2}^{*}$. Then the mapping which sends $S_{1}$ to $S_{1}^{*}, S_{2}$ to $S_{2}^{*}, R_{3}$ to $R_{3}^{\prime}$ and $R_{4}$ to $R_{4}^{\prime}$ gives an isomorphism of $\pi$ onto $\pi^{\prime}$. If $k=-k^{\prime}$, we may set $S_{i}^{\prime}=S_{i}^{*}, i=1,2, R_{3}^{\prime}=-R_{3}^{*}, R_{4}^{\prime}=R_{4}^{*}$.

Now assume that $\pi$ and $\pi^{\prime}$ are isomorphic for $k \neq \pm k^{\prime}$. Then there exists a linear transformation $\chi$ of $E^{4}$ mapping $h(\pi)$ onto itself and mapping $N$ onto $N^{\prime}$. But it is straightforward to verify that if $\chi$ maps $h(\pi)$ onto itself it must be completely reducible, mapping the invariant space of $h(\pi)$ and its orthogonal complement onto themselves. This shows that $N$ must have two distinct canonical bases $S_{1}+(k / \rho) R_{3}, S_{2}, R_{3}, R_{4}$ and $S_{1}^{\prime}+\left(k^{\prime} / \rho\right) R_{3}^{\prime}, S_{2}^{\prime}, R_{3}^{\prime}, R_{4}^{\prime}$, where $k \neq \pm k^{\prime}$. But as we have shown previously these bases must be related by a completely reducible matrix which maps $S_{1}$ and $S_{2}$ onto a linear combination of $S_{1}^{\prime}$ and $S_{2}^{\prime}$ and $R_{3}, R_{4}$ into an integer linear combination of $R_{3}^{\prime}$ and $R_{4}^{\prime}$. This is clearly impossible if $k \neq \pm k^{\prime}$.

We have now proved the following theorem.

THEOREM 8. Let $\pi$ be the fundamental group of a compact real four dimensional locally hermitian manifold. Then $\pi$ may be considered as a subgroup of $R(4)$. If $N$ is the subgroup of pure translations of $\pi$, then $\pi / N$ is a cyclic group $h(\pi)$ of order $1,2,3,4$ or 6 and $N$ has generators of the form

$$
S_{1}+(k / \rho) R_{3}, S_{2}, R_{3}, R_{4}
$$

where $R_{3}, R_{4}$ span the invariant space of $h(\pi), S_{1}$ and $S_{2}$ lie in the orthogonal complement, and $k=0$ if $\rho=1 ; k=0$ or 1 if $\rho=2 ; k=0$ or 1 , if $\rho=3 ; k=0,2$ if $\rho=4 ; k=0$ if $\rho=6$; where $\rho=$ order of $h(\pi)$. Further $\pi=\pi^{\prime}$ if and only if $h(\pi)$ $=h\left(\pi^{\prime}\right)$ and $k= \pm k^{\prime}$.

\section{APPENDIX}

Topological results.

THEOREM 9. Every locally hermitian manifold is a Kahler manifold with respect to its hermitian metric. 
It is a known theorem that the odd dimensional betti numbers of a Kahler manifold are even (see [5]).

THEOREM 10. The odd dimensional betti numbers of a locally hermitian manifold are even.

It is also well known that the odd dimensional betti numbers must be greater than or equal to one (see [5]). We will give an independent proof of this theorem for our special case.

It is now our purpose to characterize the harmonic forms on an $n$-dimensional compact locally euclidean manifold $M(\pi)$, where the fundamental group of $M(\pi)$ is $\pi$. Now Bieberbach has proved in [3] that a compact locally euclidean manifold has as covering space the $n$-dinensional torus $T^{n}$. Let $p_{1}$ be the covering map of $T^{n}$ onto $M(\pi)$, and let $\omega$ be a harmonic form on $M(\pi)$. Then $P_{1}^{*}(\omega)$ is a harmonic form on $T^{n}$, relative to the locally euclidean structure of $T^{n}$. But if $p_{2}$ denotes the covering map of $E^{n}$ onto $T^{n}$ and $\omega^{\prime}$ denotes a harmonic $r$ form on $T^{n}$ then $p_{2}^{*}\left(\omega^{\prime}\right)=\sum A_{i_{1}} \cdots_{i_{r}} d x_{i_{1}} \wedge \cdots \wedge d x_{i_{r}}$ where the $A_{i_{1}} \cdots i_{r}$ are constants and $1 \leqq i_{1}, \cdots, i_{r} \leqq n$. Hence we have shown

THEOREM 11. Let $\omega$ be a harmonic $r$ form on a compact locally euclidean space, then $\left(p_{2} \circ p_{1}\right)^{*} \omega=\sum A_{i_{1}} \ldots i_{r} d x_{i_{1}} \wedge \cdots \wedge d x_{i_{r}}$, where the $A_{i_{1}} \ldots i_{r}$ are constants.

COROLlary 4. Let $M(\pi)$ be a compact locally euclidean manifold. Then the $j$ betti number of $M(\pi)$ is less than or equal to the $j$ betti number of $T^{n}, j=1, \cdots, n$.

This is a simple consequence of the Hodge Theorem and Theorem 11. We will state and prove a slightly stronger theorem.

COROLLARY 5. Let $M(\pi)$ be an $n$-dimensional compact locally euclidean space whose $j$ betti number equals the $j$ betti number, $n \geqq j \geqq 0$, of $T^{n}$. Then $M(\pi)$ is homeomorphic to $T^{n}$.

Proof. There exist $n$ linearly independent harmonic one forms $\omega_{i}, i=1$, $\cdots, n$ on $M(\pi)$. Let $\left(p_{2} \circ p_{1}\right){ }^{*} \omega_{i}=\sum a_{i j} d x_{j}$. Hence there exists a coordinate system $\left(x_{1}^{\prime}, \cdots, x_{n}^{\prime}\right)$ on $E^{n}$ such that $\omega_{i}=d x_{i}^{\prime}, i=1, \cdots, n$ and $d x_{i}^{\prime}$ is invariant under $\pi$. Hence $\pi$ is generated by $n$ linearly independent translations. This proves the corollary.

COROllary 6. The even dimensional betti numbers of a compact $2 n$-dimensional locally hermitian manifold are greater than or equal to one.

Proof. $d s^{2}=\sum_{j} d z_{j} d \bar{z}_{j}$. Hence the associated quadratic form is $\Omega=d x_{1}$ $\wedge d x_{2}+\cdots+d x_{2 n-1} \wedge d x_{2 n}$. But this is harmonic and $\Omega^{i} \neq 0 j=1, \cdots, n$. This proves the corollary.

COROLlaRy 7. Let $M(\pi)$ be a $2 n$-dimensional compact locally hermitian manifold. Then $1+\sum_{j=1}^{n} p_{2 j} \equiv 0 \bmod (4)$ where $p_{2 j}$ denotes the $2 j$ betti number, $j=1, \cdots, n$. 
Proof. Since the Euler characteristic of $M(\pi)$ is zero and $n$ is even $1+\sum_{j=1}^{n} p_{2 j}=2 \sum_{j=1}^{n / 2} p_{2 j-1}$ by the Poincaré Duality Theorem. But $p_{2 j-1}$ must be even by Theorem 5 . This completes the proof of the corollary.

TheOREM 12. A compact four dimensional locally euclidean space is locally hermitian if and only if it is a torus or has Poincare polynomial $1+2 x+2 x^{2}$ $+2 x^{3}+x^{4}$.

Proof. Assume $M(\pi)$ is locally hermitian and let $1+a_{1} x+a_{2} x^{2}+a_{1} x^{8}+x^{4}$ be its Poincaré polynomial. Then $a_{2}+2 \equiv 0 \bmod (4)$. Hence $a_{2}=2$ or 6 . Hence the only possible Poincaré polynomials are

$$
1+2 x+2 x^{2}+2 x^{3}+x^{4} \text { and } 1+4 x+6 x^{2}+4 x^{3}+1 .
$$

But Corollary 4 proves that if $M(\pi)$ has Poincaré polynomial $1+4 x+6 x^{2}$ $+4 x^{3}+1$ it is a torus.

Now let $M(\pi)$ be compact, locally euclidean and have Poincaré polynomial $1+2 x+2 x^{2}+2 x^{8}+x^{4}$. Then there exist two linear independent harmonic one forms $\omega_{1}^{\prime}$ and $\omega_{2}^{\prime}$ on $M(\pi)$. Hence we may choose new harmonic forms $\omega_{1}$ and $\omega_{2}$ which are orthogonal and linearly independent and lie in the space spanned by $\omega_{1}^{\prime}$ and $\omega_{2}^{\prime}$. We may choose a new coordinate system $x_{1}, \cdots, x_{4}$ in $E^{4}$ such that $d x_{1}=\omega_{1}$ and $d x_{2}=\omega_{2}$. With respect to this new basis each element of $\pi$ has a matrix representation of the form

$$
\left(\begin{array}{ccccc}
1 & 0 & 0 & 0 & a_{1} \\
0 & 1 & 0 & 0 & a_{2} \\
0 & 0 & a_{33} & a_{34} & a_{3} \\
0 & 0 & -a_{34} & a_{33} & a_{4} \\
0 & 0 & 0 & 0 & 1
\end{array}\right) .
$$

Hence $\pi \subset R(n, C)$. Hence $M(\pi)$ is locally hermitian with respect to this basis.

\section{REFERENCES}

1. L. Auslander and M. Kuranishi, On the holonomy groups of locally euclidean spaces, to appear in Ann. of Math.

2. L. Auslander and L. Markus, Holonomy of flat affinely connected manifolds, Ann. of Math. vol. 62 (1955) pp. 139-152.

3. L. Bieberbach, Über die Bewegungsgruppen der Euklidische Raïme, Math. Ann. vol. 70 (1911) pp. 297-336.

4. - Über die Bewegungsgruppen der Euklidische Railme (Zweite Abhandlung), Math. Ann. vol. 72 (1912) pp. 400-412.

5. B. Eckmann and $H$. Guggenheimer, Sur les varietes closes d métrique hermitiènne sans torsion, C. R. Acad. Sci. Paris vol. 229 (1949) pp. 505-506.

6. W. Hantzsche and H. Wendt, Dreidimensionale euklidische Raumformen, Math. Ann. vol. 110 (1934-1935) pp. 593-611.

INSTITUTE for AdVANCEd StUdy,

Princeton, N. J. 